\title{
A Rare Case of Maxillary Sinus Cancer that involve the Orbit and adjacent Structures
}

\begin{abstract}
43year old male diagnosed as case of Maxillary sinus cancer that involved the orbit. CT scan of face revealed large ill defined extensively infiltrated soft tissue mass of $7^{\star} 5^{\star} 4.4 \mathrm{~cm}$ in maxillary sinus which extended to infratemporal fossa and erosion of floor of the right orbit with infiltration of right inferior rectus muscle. The prognosis of maxillary sinus cancer with involvement adjacent structures is not very good (usually diagnosed at an advanced stage), so to attain an improved result it was essential to secure adequate resection of margins. So we followed a standard treatment protocol that was total maxillectomy with orbital exenteration, followed by postoperative radiation therapy.
\end{abstract}

Keyword : Maxillary Sinus, Carcinoma, orbital floor, Craniofacial resection, reconstruction.

\section{Introduction:}

Cancer of the maxillary sinus is relatively uncommon constituting $0.2 \%$ to $2.2 \%$ of all malignancies, $5 \%$ of all malignant aerodigestive tumours and $80-90 \%$ of all Paranasal sinus tumours (Ackerman and del Regato 1985)[1]. Most patients with maxillary sinus cancer have no symptoms in the early stage and, therefore, many of these patients are diagnosed in the advanced stage of the disease. Local extension of these tumours results in invasion of the skull base, and this is usually managed by craniofacial resection (CFR) with postoperative adjuvant radiotherapy[2].

Tumors may invade the orbit via preformed pathways, via neurovascular structures, or by direct extension through bone. The term "orbital exenteration" is normally applied to complete removal of the contents of the orbit, including the eyelids. This is rarely required for most sinonasal tumors. "Orbital clearance" is a procedure in which the globe, muscles, fat, and periorbita are removed, while the lids, and usually the palpebral conjunctiva, are preserved[3].

\begin{tabular}{|l|l|}
\hline \multicolumn{2}{|c|}{ Access this article online } \\
\hline \multirow{2}{*}{$\begin{array}{l}\text { Website: } \\
\text { www.ujds.in }\end{array}$} & Quick Response Code \\
\hline $\begin{array}{l}\text { DOI: } \\
\text { https://doi.org/10.21276/ujds.2020.6.3.16 }\end{array}$ \\
\hline
\end{tabular}

The reason for undertaking such an aggressive and disfiguring operation is that all other (eye-sparing) options would either jeopardize the adequacy of the excision or compromise the long-term viability of the eye[4].

\section{Case Report :}

We presented a case of a patient 43 old man, a farmer by profession and resident of Fatehpur district, with a history of right hemi facial swelling from past 15 days with nasal discharge and diplopia (blurred vision) since 5 days with no significant medical history, patient gave history of gutkha chewing since 5 Years with frequency of 8-10 pouches in a day and history of beedi smoking since 8 to 10 years. Patient quit his habit of tobacco chewing and beedi smoking one month back.

\section{${ }^{1}$ KUNDU, A., ${ }^{2}$ YADAV, S. S., ${ }^{3}$ KUMAR, V., ${ }^{4}$ VATS, A. \\ ${ }^{1-4}$ Rama Dental College and Hospital, Kanpur}

Address for Corresponding : Dr. Sardar Singh Yadav Rama Dental College and Hospital, Kanpur

Email : sardar1.man@gmail.com

Received : 14 Nov. 2020, Published : 31 Dec. 2020

How to cite this article: Kundu, A., Yadav, S. S., Kumar, V., \& Vats, A. (2021). A rare case of maxillary sinus cancer that involve the orbit and adjacent structures. UNIVERSITY JOURNAL OF DENTALSCIENCES, 6(3). 88-91 

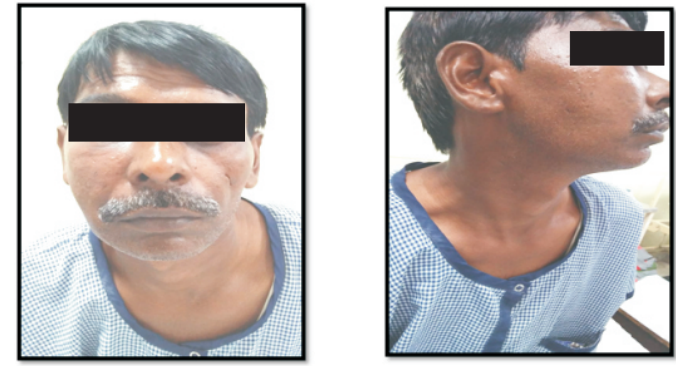

Fig.1: Patient clinical pictures showing swelling over right side of face no clinical lymph node seen over neck.

Patient also gave history of extraction by a dentist 2 months back since then the patient had a complaint of food particles and liquid coming out from his nose (Oro-Antral fistula) during food intake.

The patient was then advised for a Biopsy and CT FACE and NECK with contrast, punch biopsy was taken from the right maxillary Antrum and sent for Histopathological examination which revealed that there was presence of well differentiated Squamous Cell Carcinoma of the same.

CT scan of face was done which revealed maxillary antrum carcinoma (large ill defined extensively infiltrated soft tissue mass of $7 * 5 * 4.4 \mathrm{~cm}$ ) which extends to infratemporal fossa. Coronal view of maxilla showing the infiltrating soft tissue mass into the antrum that's extending medially to the nasal fossa and adjacent nasal pharynx on the right side and superiorly erosion of floor of the right orbit with infiltration of right inferior rectus muscle. General Physical Examination and Systemic Examination were normal. Chest X-rayposterior anterior (electrocardiogram, complete hemogram, Random blood sugar, Liver function test, Renal Function Test and viral Markers were done and all reports were within normal limits.

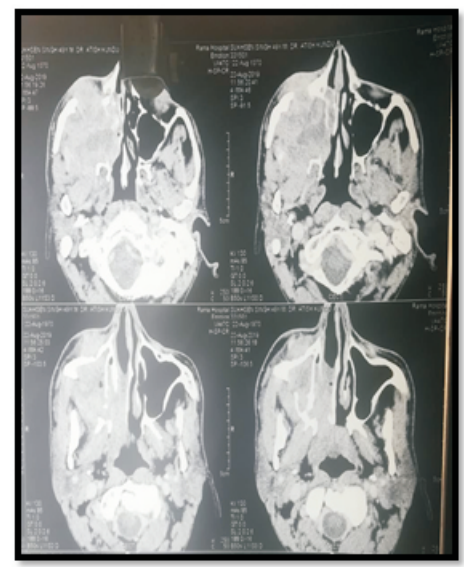

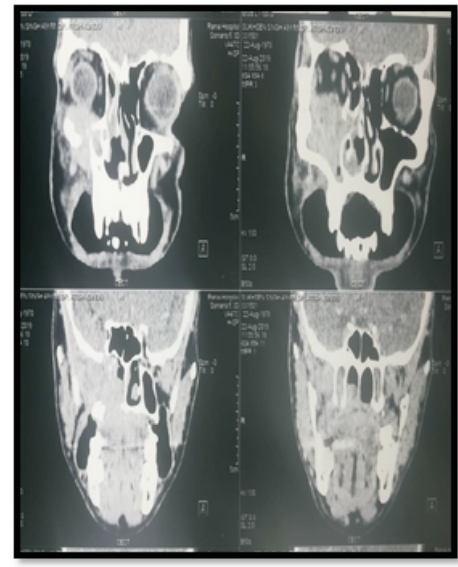

Fig. 2 : CT scan face axial and coronal view large ill defined extensively infiltrated soft tissue mass of $7 * 5 * 4.4 \mathrm{~cm}$ which extends to infratemporal fossa and superiorly erosion of floor of the right orbit with infiltration of right inferior rectus muscle.

TNM staging is T4a N0 M0 according to AJCC 8th edition on the basis of size and extent of primary tumor. This tumor was managed by Total Maxillectomy with orbital Exenteration and Modified neck dissection, Reconstruction with radial forearm free flap. Counseling of the patient was done in presence of his family member and explained about the risks and benefits of surgery. Written informed consent was taken for surgery, general anaesthesia, scar and eye removal.

Patient placed in supine position and general anaesthesia was introduced with nesotracheal intubation from left nostril. Paint and drape was done. Neck marking according to schobinger done and subplatysmal flap was raised all fibrofatty tissue was removed with level-I to Level-V. Sternocleidomastoid muscle was sacrificed and accessory spinal nerve was preserved. Facial artery and vein was preserved for anastamosis. External carotid artery was clamped by Bulldog clamp. A modified Webber Fergusson incision was made and it was connected to the vestibular degloving incision, the whole surface of maxilla and maxillary process was exposed. The eyeball was removed along with orbital contents and ophthalmic artery was ligated. Simultaneously bony cuts were made at the level of the right frontozygomatic suture temporal process of zygomatic bone frontal process of maxilla along with lacrimal bone Lateral nasal bone and extended up mid line, Palatal Osteotomy and Osteotomy to separate maxillary tuberosity from pterygoid plate. Whole maxilla was removed with the orbital contents and sent for the frozen biopsy and all tumor margins free from diseases. Radial free fore arm flap was harvested from right 
arm and cover the defect (palatal and nasal covering. Orbital cavity cover eyelid skin flap. After 3 weeks of surgery we Plan eye prosthesis that is connected to spectacles.
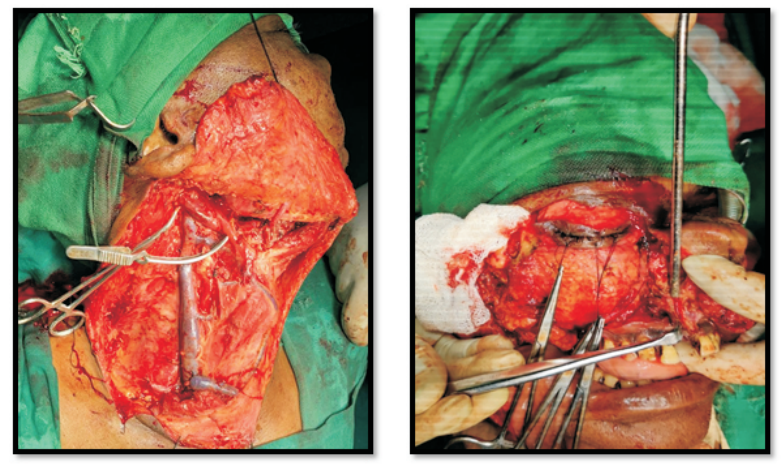

Fig. 3 : MND Type-II neck dissection and modified Webber Fergusson incision was made and it was connected to the vestibular degloving incision, the whole surface of maxilla and maxillary process was exposed.

Final Histopathological report of the specimen (En Bloc resected tumor and fibro fatty tissue of neck) revealed that all margins were free from cancer and no regional lymph node metastasis was seen.
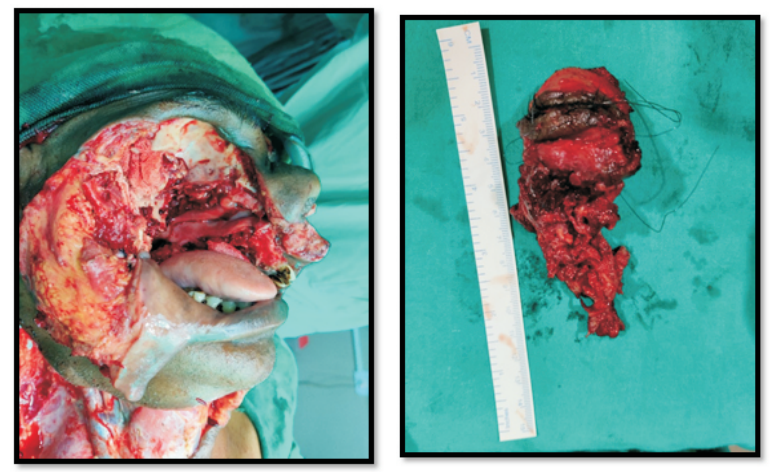

Fig.4 : Simultaneously bony cuts were made- En bloc maxillectomy with orbital exenteration done and tissue send for the frozen biopsy.

\section{Discussion}

The presence of large air spaces within the paranasal sinus probably allows the asymptomatic expansion of sinus carcinoma5. Common symptoms include pain, nasal discharge, epistaxis and obstruction, commonly affecting males more than females at a ratio of 1.5:1.1 Other symptoms include: (1) nasal fullness, stuffiness or obstruction, (2) pain, (3) cheek paresthesia, (4) cheek fullness or swelling, (5) palatal bulge, (6) persistent, non-healing nasal/oral sore or ulcer, (7) nasal mass, (8) proptosis, diplopia or lacrimation6.
Early symptoms differ little from common nasal complaints and their temporary regression by antibiotics falsely reassures both the patient and the physician. When more alarming symptoms, such as ocular complaints, cranial nerve deficits, or cheek mass, are apparent, the outcome tends to be less favorable[5].

Maxillary sinus carcinoma presents a therapeutic challenge to both the surgeon and the radiation oncologist. Because symptoms are vague and nonspecific, the majority of carcinomas are diagnosed as locally advanced disease [2]. Extension to contiguous structures including the orbit, ethmoid sinus, sphenoid sinus, nasal cavity, nasopharynx, pterygoid fossa, palate and cheek may occur and can be a potential problem in the surgical and/or radiotherapeutic management of this disease[7].

In advance horizons of oral and maxillofacial surgery few surgery are destructive or unpleasant to perform as total maxillectomy with orbital exenteration. Ample reconstructive procedure are present to cover the orbital cavity ; the temporalis muscle pedicled flap, the galea fascia or pericranial flap, the myocutaneous pedicled flap and the revascularized free flap[8].

Management of tumours of maxillary antrum needs a multidisciplinary approach. Surgery and radiation, alone or in combination are the mainstay of treatment. Majority of the patients are already advanced at the time of presentation and either of the modalities used alone gives very poor results, 5 year survivals being in range of $10-15 \%$ only[1].

The status of the surgical margins was an important predictor for overall, disease-specific, and recurrence-free survival. Complete surgical excision with histologic negative margins of resection should be one of the main goals of craniofacial resection for malignant paranasal sinus tumors[2].

With properly planned combined approaches, 5 year survival has improved from 10-29\% (Mc Nicoll et al 1984) to 50-70\% (Lewis 1972, Lee et al 1981. Parsons et al 1987). This improvement has resulted from better management and control of primary (Shidnia et al, 1984). Local recurrence rate is markedly less with combined modality approach than with radiation alone[1].

A study by Won et al.[5] concluded that induction chemotherapy (ICT) in locally advanced maxillary sinus cancers increased the possibility of tumor down-staging and 
complete resection with orbital preservation. One of four different treatment modalities, including intra-arterial (IA)ICT, IV-ICT, concurrent chemoradiotherapy and surgical resection, was selected as a primary treatment based on the TNM stage, performance status, age and comorbidity. Although there were no significant differences in response rate and toxicity profile between the two groups of ICT, IAICT was superior to IV-ICT with respect to tumor down staging and local tumor contro[9].

\section{Conclusion:}

Cancer of the maxillary sinus that's involves the orbital contents is relatively rare and diagnosed in advance stage. Complete surgical excision with histologic negative margins of resection is an important determinant of survival and local control. Surgery with radiation is a better treatment option.

\section{References:}

1. Sharma s. et al. Carcinoma of the maxillary antrum - a 10 year experience, Indian Journal of Otolaryngology; Volume 43, No. 4, December 1991

2. Ganly I. Craniofacial resection for malignant paranasal sinus tumors: report of an international collaborative study 11 April 2005 in Wiley InterScience ( w w w. in terscience.wiley.com) DOI: 10.1002/hed.20165.

3. Sua'rez c. et al. Management of the orbit in malignant sinonasal tumors, 19 November 2007 in Wiley InterScience (www.interscience.wiley.com) DOI: 10.1002/hed.20736.

4. Hoffman G R, Jefferson N D, Reid C B, and Leonard R, Orbital Exenteration to Manage Infiltrative Sinonasal, Orbital Adnexal, and Cutaneous Malignancies Provides Acceptable Survival Outcomes: An Institutional Review, Literature Review, and Meta-Analysis, J Oral Maxillofac Surg 74:631-643, 2016.

5. Dulguerov P, Michael S. Jacobsen, Allal A S, Lehmann W, Calcaterra T, Nasal and Paranasal Sinus Carcinoma: Are We Making Progress?A Series of 220 Patients and a Systematic Review, American Cancer Society August 30, 2001 .

6. Hernandez AK, Cabungcal AC, Maxillary Sinus Squamous Cell Carcinoma in a Tertiary Hospital in the Philippines, Philippine Journal Of Otolaryngology-Head and Neck Surgery Vol. 34 No. 1 January - June 2019.

7. Ashraf M, Biswas J, Dam A, Bhowmick A, Jha JK, Sing V, Nayak S, Results of Treatment of Squamous Cell
Carcinoma of Maxillary Sinus: A 26-Year Experience, World J Oncol. 2010;1(1):28-34

8. A. Croce, A. Moretti, L. D Agostino, P. Zingariello, Orbital exenteration in elderly patients: personal experience, ACTA otorhinolaryngologica ita lica 2008;28:193-199.

9. Sarkar SK, Chaudhuri A, Banerjee C, Banerjee S, Primary antral carcinoma managed by en-bloc radical maxillectomy with orbital exenteration, Journal of the Scientifi c Society, Vol 41 / Issue 3 / SeptemberDecember 2014. 